\title{
Full-model wavenumber inversion: An emphasis on the appropriate wavenumber continuation
}

\author{
Tariq Alkhalifah ${ }^{1}$
}

\begin{abstract}
A model of the earth can be described using a Fourier basis represented by its wavenumber content. In full-waveform inversion (FWI), the wavenumber description of the model is natural because our Born-approximation-based velocity updates are made up of wavefields. Our objective in FWI is to access all the model wavenumbers available in our limited aperture and bandwidth recorded data that are not yet accurately present in the initial velocity model. To invert for those model wavenumbers, we need to locate their imprint in the data. Thus, I review the relation between the model wavenumber buildup and the inversion process. Specifically, I emphasize a focus on the model wavenumber components and identified their individual influence on the data.
\end{abstract}

Missing the energy for a single vertical low-model wavenumber from the residual between the true Marmousi model and some initial linearly increasing velocity model produced a worse least-squares fit to the data than the initial model itself, in which all the residual model wavenumbers were missing. This stern realization validated the importance of wavenumber continuation, specifically starting from the low-model wavenumbers, to higher (resolution) wavenumbers, especially those attained in an order dictated by the scattering angle filter. A numerical Marmousi example determined the important role that the scattering angle filter played in managing the wavenumber continuation from low to high. An application on the SEG2014 blind test data set with frequencies lower than $7 \mathrm{~Hz}$ muted out further validated the versatility of the scattering angle filtering.

\section{INTRODUCTION}

Seismic data are inherently dependent on the short-wavelength components of the earth model for the presence of the majority of the recorded events, and they are dependent on the long-wavelength components for the geometrical shapes of such events. We usually use the geometrical features of the wavefield to extract the long-wavelength components first, which are in turn used to place the scattered events in their accurate positions in what we typically refer to as imaging. Likewise, in linearized inversion, we use the extracted smooth model from analyzing the geometrical behavior of the wavefield (using, for example, migration velocity analysis [MVA]) to iteratively place the perturbations in their accurate locations in the velocity model. Alternatively, we can obtain background velocity information from the data, if sufficiently low frequencies are available, in an iterative process in what we refer to as frequency continuation in full-waveform inversion (FWI; Bunks et al., 1995;
Pratt et al., 1996; Virieux and Operto, 2009). The key element in the inversion process, using either the geometrical features of the wavefield or low frequencies, is the model wavenumber buildup strategy. Thus, FWI conventionally requires that every model wavenumber component is placed in the model within a half cycle of its true location, and such placement is usually controlled by the lower wavenumbers. For a certain, initially inaccurate, propagator (background) velocity, this can be achieved if the injected model wavenumber is low. These added low wavenumbers tend to improve the propagator velocity, so that higher wavenumbers may meet the threshold of a half cycle. From the data stand point, the wavenumber continuation is commonly controlled by inverting first for low frequencies and large offset-to-depth ratios (Bunks et al., 1995; Pratt et al., 1996; Virieux and Operto, 2009; achieved in one form by damping later arrivals; Jang et al., 2009).

Recently, many have explored the wavenumber continuation theme from the model stand point, instead of the data (Albertin et al.,

\footnotetext{
Manuscript received by the Editor 9 October 2015; revised manuscript received 3 December 2015; published online 06 April 2016.

${ }^{1}$ King Abdullah University of Science and Technology, Physical Sciences and Engineering, Thuwal, Saudi Arabia. E-mail: tariq.alkhalifah@kaust.edu.sa; tkhalfah@kacst.edu.sa.

(C) 2016 Society of Exploration Geophysicists. All rights reserved.
} 
2013; Almomin and Biondi, 2013; Tang et al., 2013), specifically, by conditioning and filtering the gradient. Despite the value of data decimation and selection, the real objective of this process is far more apparent in the model domain and specifically at the gradient level (Sirgue and Pratt, 2004). Such developments resulted in a more direct control of the model wavenumber update, independent of frequency, given by the scattering angle filter (Alkhalifah, $2014 b$ ). The scattering-angle filtering provided a directionally independent filter (with respect to dip) of the gradient valid for FWI and MVA gradients. Unlike a classic smoothing operator, the scattering angle filter is governed by a physical quantity of the scattering process providing low wavenumbers along even complex raypaths, resulting from a complex background velocity (such as having salt bodies). This is provided by its velocity-dependent smoothing operator, more easily implemented in the wavenumber domain and an extended normalized time-lag axis. However, proper wavenumber continuation also requires the proper extraction of such model wavenumbers from the data. To do so, we need to use data-based inversion, as well as those that rely on the image (Fleury and Perrone, 2012; Ma et al., 2012; Xu et al., 2012; Almomin and Biondi, 2013; Wang et al., 2013; Alkhalifah and Wu, 2016). Low-wavenumber information extracted directly from the data residual typically has mild depth penetration limited by the penetration of the diving waves. However, we can also extract low wavenumbers from the data by imaging the reflections. We can either use extended images for classic MVA (Al-Yahya, 1989; Symes and Kern, 1992; Plessix et al., 1995; Clement et al., 2001) or apply a demigration to implement what is referred to as reflection-waveform inversion (RWI; Chavent and Plessix, 1999; Clement et al., 2001).

The most influential model wavenumbers in the data are the zero to low wavenumbers. On the other hand, the influence of the high wavenumbers is localized in the data as scattering, yet FWI has a hard time inverting for the low wavenumbers because the update process (the linear form) is based on scattering theory. The unwrapped phase of the depth model wavenumber allows us to move the influence of low wavenumbers to the sensitivity range of FWI (Alkhalifah, 2014a). Often missing in such arguments is a reasonable account of the true impact of various model wavenumbers in the data, and conversely, the source of such model wavenumbers within the data considering the multitude of inversion techniques available, including those that use the image. In this paper, I review the model wavenumber linearized relation to the data, and I analyze the extracted wavenumbers that we may obtain from image-based methods, such as RWI, and specifically, from classic FWI. We will then look at the scattering angle filter as an approach to control such wavenumbers regardless of their source. An application of the FWI based on the scattering angle filter on the SEG2014 data set will highlight some of its features.

\section{FWI AND RWI GRADIENTS}

Although FWI inverts for the background velocity (low-wavenumber components) using direct and diving waves, RWI inverts for the background velocity along the reflection wavepaths. In addition, FWI and RWI try to predict single and double scattering locations, respectively. With low enough frequencies, the background and scattering components in both cases could exert the proper continuation in wavenumbers. For FWI, this continuation, as we will see later, is dominated by the vertical wavenumber component. For RWI, such continuation is dominated by the near-horizontal wavenumber component due to the vertical wavepath. However, with dipping reflectors, we can have continuations between the RWI-produced and the FWI-produced wavenumbers. To understand the role of RWI and FWI, let us look into their gradients.

Starting from the definition of the classic FWI objective function given by Tarantola (1984)

$$
E_{\mathrm{FWI}}(m)=\sum_{i}\left|d_{o_{i}}-d_{s_{i}}(m)\right|^{2}
$$

where $i$ is the source index, $d_{o}$ is the observed data, $d_{s}=$ $u_{s}(x, y, z=0, t)$ is the modeled synthetic data, and $m(\mathbf{x})$ is (any form of) the velocity model described in space using the vector $\mathbf{x}=\{x, y, z\}$, along the Cartesian coordinates, with $t$ as time. From this point on, and for simplicity, but without loss of generality, I represent wavefields and data in the frequency domain. In this case, the modeled wavefield $u_{s}$ satisfies the following wave equation:

$$
L(m) u_{s_{i}}=f(\omega) \delta\left(\mathbf{x}-\mathbf{x}_{s_{i}}\right),
$$

for a particular source location $\mathbf{x}_{s_{i}}$, and $f$ is the source function given in the frequency domain with

$$
L(m)=L^{*}(m)=\nabla^{2}+\omega^{2} m(\mathbf{x}),
$$

where $L$ is the Helmholtz operator, which in this case is self-adjoint, $\omega$ is the angular frequency, and $\nabla^{2}$ is the Laplacian operator. The gradient corresponding to such an objective function is given by (Tarantola, 1984)

$$
R_{\mathrm{FWI}}(\mathbf{x})=\omega^{2} \sum_{i} u_{s_{i}}(\mathbf{x}) u_{r 1_{i}}(\mathbf{x})
$$

with

$$
L(m) u_{r 1_{i}}=\sum_{j} \delta\left(\mathbf{x}-\mathbf{x}_{r_{j}}\right)\left(d_{o_{i j}}-d_{s_{i j}}\right)^{*},
$$

where $\mathbf{x}_{r_{j}}$ are the locations of the receivers, and $j$ is the receiver index.

On the other hand, we can produce data directly from an image and use the following objective functional (Clement et al., 2001):

$$
E_{\mathrm{RWI}}(m, I)=\sum_{i}\left|d_{o_{i}}-d_{m_{i}}(m, I)\right|^{2},
$$

where $d_{m}$ is the (Born approximation) modeled data from the image. The image $I(\mathbf{x})$ satisfies

$$
I(\mathbf{x})=\sum_{i} u_{s_{i}}(\mathbf{x}) u_{a_{i}}(\mathbf{x}),
$$

where the receiver wavefield $u_{a_{i}}$ is given by 


$$
L^{*}(m) u_{a_{i}}=\sum_{j} \delta\left(\mathbf{x}-\mathbf{x}_{r_{j}}\right) d_{o_{i j}}^{*}
$$

and thus

$$
L(m) \delta u_{s_{i}}=I(\mathbf{x}) u_{s_{i}}(\mathbf{x})
$$

and $d_{m_{i}}=\delta u_{s_{i}}\left(\mathbf{x}_{r_{i}}\right)$. In this case, to obtain mainly low-wavenumber gradients along the wavepath from RWI, $d_{m_{i}}$ should match $d_{o_{i}}$ at zero offset, or in other words, we should use true amplitude or leastsquares migration to obtain $I(\mathbf{x})$. As Alkhalifah and $\mathrm{Wu}$ (2016) show, such least-squares migration should focus only on the near-zero-offset data.

The gradient corresponding to this objective functional is given by (Clement et al., 2001)

$$
R_{\mathrm{RWI}}(\mathbf{x})=\omega^{2} \sum_{i}\left(u_{s_{i}}(\mathbf{x}) \delta u_{r_{i}}(\mathbf{x})+u_{r 2_{i}}(\mathbf{x}) \delta u_{s_{i}}(\mathbf{x})\right),
$$

where

$$
L(m) u_{r_{i}}=\sum_{j} \delta\left(\mathbf{x}-\mathbf{x}_{r_{j}}\right)\left(d_{o_{i j}}-d_{m_{i j}}\right)^{*}
$$

and

$$
L(m) \delta u_{r_{i}}=I(\mathbf{x}) u_{r_{i}}(\mathbf{x}) .
$$

Using the perturbed wavefield $\delta u_{s_{i}}$, we can predict part of the double scattering image by correlating this wavefield with the receiver wavefield $u_{a_{i}}(\mathbf{x})$. The other part is obtained from crosscorrelating $\delta u_{r_{i}}$ with $u_{s_{i}}$. In other words, such an image is equal to $\left(R_{\mathrm{RWI}}(\mathbf{x})\right) / \omega^{2}$. This image can serve as a source for double-scattered energy and data. This will allow us to invert for the double scattering information including the low-wavenumber components given by the wavepath between two scatterers.

\section{THE MODEL WAVENUMBER}

In FWI, the model wavenumber vector describes the resolution and dip of a potential scattering point. Based on the adjoint Born scattering approximation, which involves the interaction of the two locally planer wavefields using a crosscorrelation process (often used to obtain a velocity gradient), the resulting wavenumber vector is given by (Miller et al., 1987; Jin et al., 1992; Thierry et al., 1999)

$$
\mathbf{k}_{\mathbf{m}}=\mathbf{k}_{\mathbf{s}}+\mathbf{k}_{\mathbf{r}}=2 \frac{\omega}{v} \cos \frac{\theta}{2} \mathbf{n},
$$

which depends on, among other things, the angular frequency $\omega$ with a direction guided by a unit vector $\mathbf{n}$ normal to a potential reflector. Here, $\mathbf{k}_{\mathbf{s}}$ and $\mathbf{k}_{\mathbf{r}}$ are the source and receiver (or any state and adjoint state) wavefield wavenumber vectors, respectively, at the model point, with $\theta$ as the angle between these vectors (the scattering angle), and $v=1 /(\sqrt{m})$ is the velocity at the model point.

This relation holds for interaction between any two wavefields including single-scattered wavefields, such as in RWI. For monochromatic wavefields of $10 \mathrm{~Hz}$ frequency from each of the source and receiver for a background linearly increasing velocity model, the FWI gradient is given in Figure 1a. We refer to this function as the sensitivity kernel. The wavenumber magnitude and direction corresponding to this gradient is shown in Figure 1b. Along the diving wavepath between the source and receiver, the wavenumber tends to zero. Otherwise, in the region between the source and receiver, the wavenumber vector points vertically. Therefore, most of the resolution in the model for this setup is in the vertical direction. For the RWI case, we plot the source side (Figure 2a) and the receiver side (Figure $2 b$ ) gradients separately for a point scatterer in the middle near the bottom of the model. The gradients of the source and receiver sides are added to the update, so the wavenumbers corresponding to each of them are actually independent. As expected, the model wavenumber vector points mostly in a near-horizontal direction especially around the wavepath.

\section{FROM MODEL WAVENUMBERS TO DATA}

To search where in the data space we can extract the model wavenumbers, let us examine the data sensitivity (or specifically calculate the objective function) to specific wavenumbers in the model. I will use the Marmousi model for this experiment as shown in Figure 3a. I first consider a potential initial velocity model given by a velocity linearly increasing with depth given by $v_{0}(z)=$ $1500+z \mathrm{~m} / \mathrm{s}$, as shown in Figure 3b. The difference between the initial velocity and the true velocity is the residual $(\Delta v)$ velocity that we hope to extract from FWI (and RWI). We will first investigate the sensitivity of the objective function to the absence of cer-
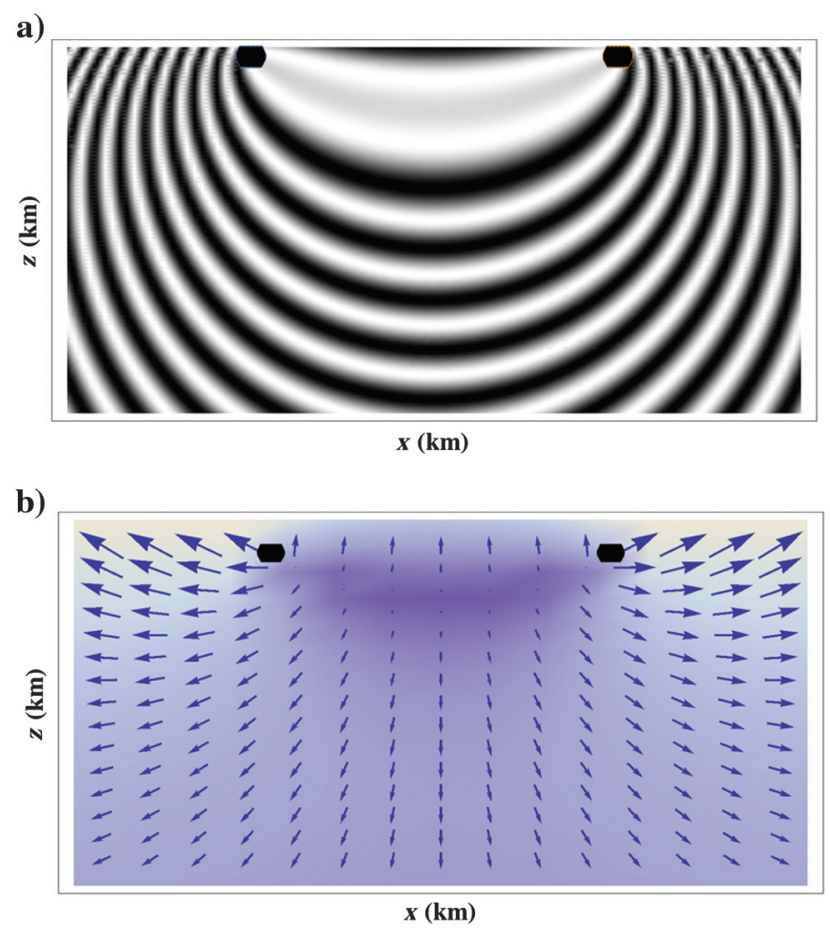

Figure 1. (a) A density plot of the gradient for a source and receiver given by the black dots for monochromatic wavefields (10 Hz frequency) corresponding to a standard FWI. (b) The model wavenumber vector magnitude and direction of the sensitivity kernel. 

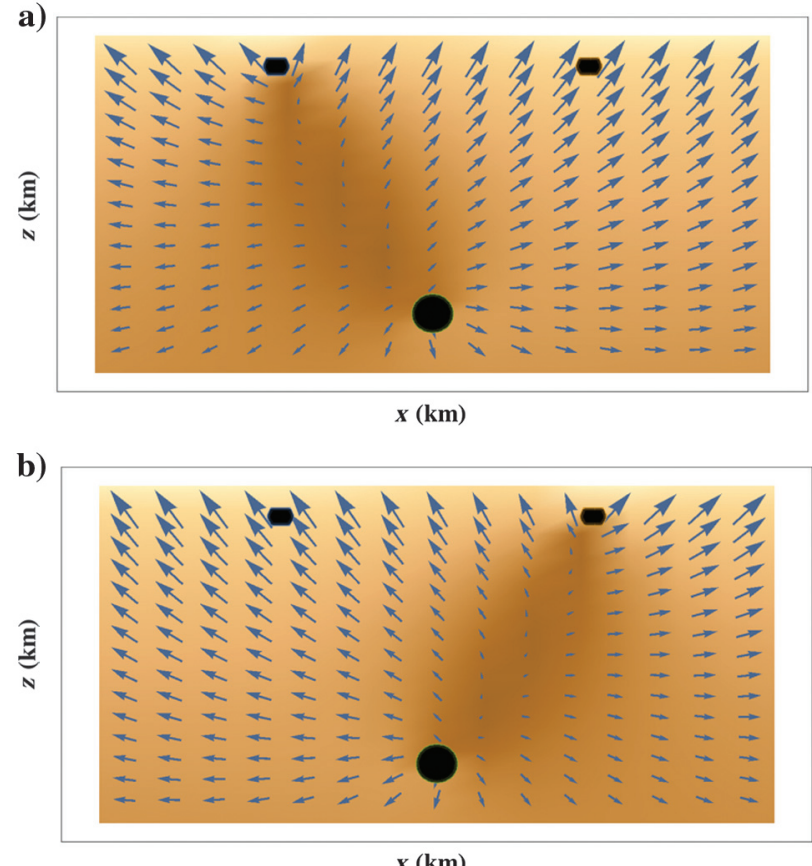

Figure 2. (a) The model wavenumber magnitude and direction for the source side of the RWI gradient for monochromatic wavefields. (b) The model wavenumber magnitude and direction for the receiver side of the RWI gradient for monochromatic wavefields.
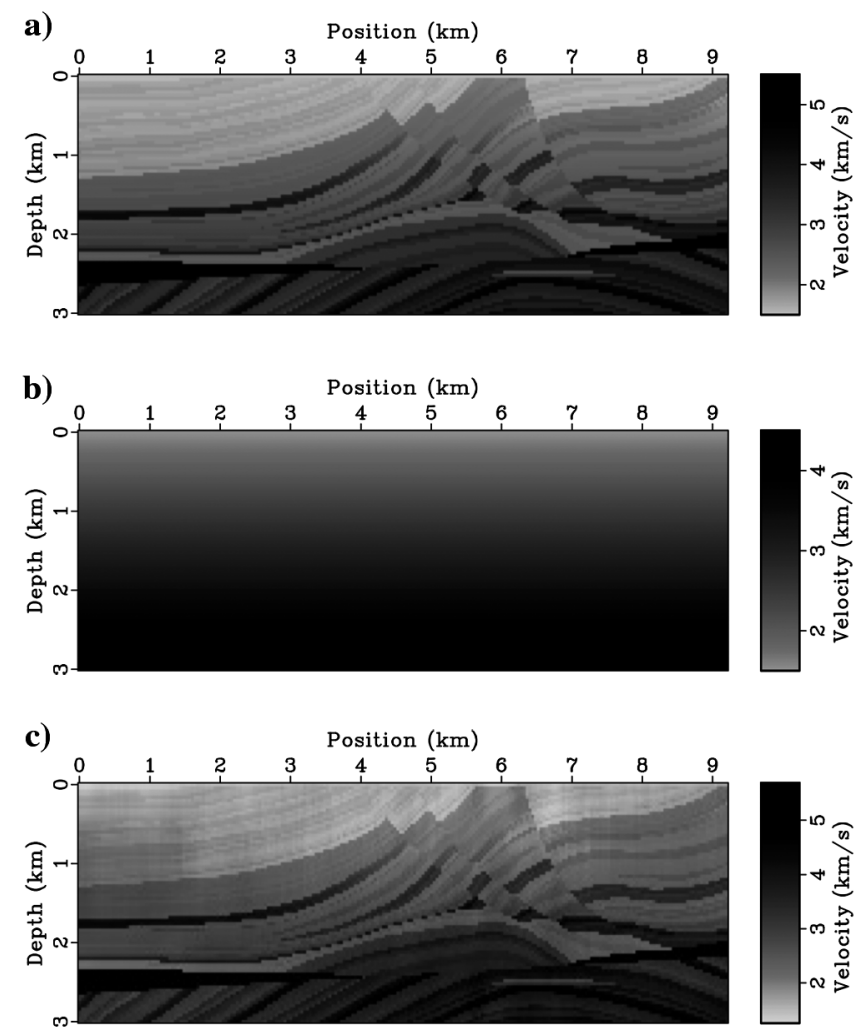

Figure 3. (a) The Marmousi velocity model sampled at $0.04 \mathrm{~km}$ literally and vertically. (b) A linearly increasing velocity model $v_{0}(z)=1.5+z \mathrm{~km} / \mathrm{s}$. (c) A velocity model constructed from muting the energy corresponding to the wavenumber $0.33 \mathrm{~km}^{-1}$ from the residual between the Marmousi model and $v_{0}(z)$. tain vertical wavenumbers in this residual. Thus, Figure $3 \mathrm{c}$ shows the Marmousi velocity model after removing the energy corresponding to the vertical wavenumber sample $0.33 \mathrm{~km}^{-1}$ (or $3 \mathrm{~km}$ wavelength). The coarsely gridded model is represented by 39 discrete depth model wavenumbers. Evidence of the lack of this wavenumber from the residual $\left(\Delta V\left(k_{z}\right)-\Delta V(0.33)\right.$, where $\Delta V$ is the vertical Fourier transform of $\Delta v)$ part can be somewhat seen in the model (Figure 3c), as long wavelength variations. Figure $4 \mathrm{a}-4 \mathrm{c}$ shows the shot gathers generated for a source located at $5600 \mathrm{~m}$ corresponding to the velocity models shown in Figure $3 a-3 c$,
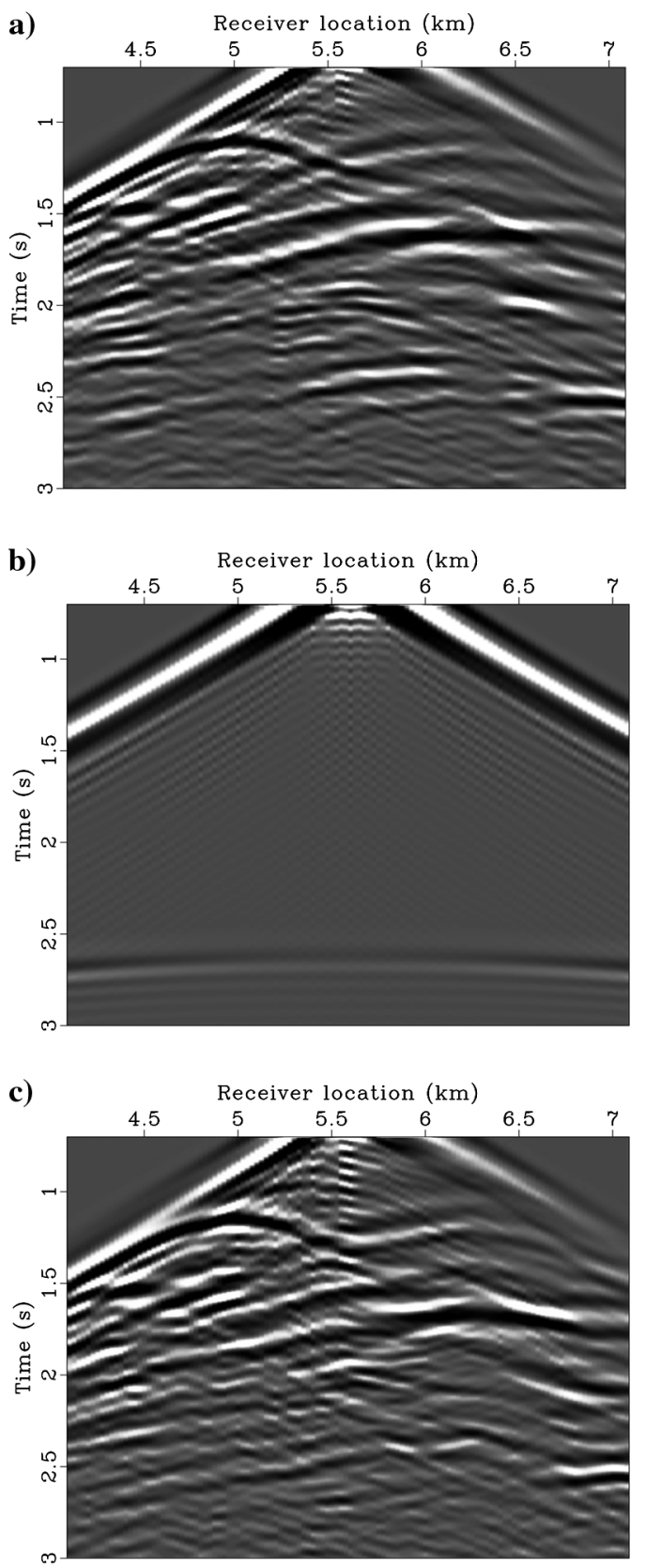

Figure 4. Shot gathers corresponding (a) to the original Marmousi model shown in Figure $3 a$, (b) to the linearly increasing velocity model shown in Figure 3b, and (c) to the model shown in Figure 3c. Shot surface location is $5.6 \mathrm{~km}$. 
respectively. The source is given by a Ricker wavelet with a peak frequency of $10 \mathrm{~Hz}$, with a spectrum, shown in Figure 5a (dotted curve), mapped to wavenumber using a (representative) velocity of $3 \mathrm{~km} / \mathrm{s}$. Figure $5 \mathrm{~b}$ (solid) shows the objective function given by the least-squares fit for models, in which $\Delta v$ is missing the energy corresponding to the wavenumber given by the horizontal axis, and compared with the objective function (dashed curve) for the linear velocity model in Figure $3 \mathrm{~b}$, all normalized by the maximum objective value for these models. The shot gather emerging from the model with the missing $0.33 \mathrm{~km}^{-1}$ vertical wavenumber (Figure 4c) looks far more similar to the true Marmousi shot gather (Figure 4a) than that produced from the linear velocity model (Figure $4 \mathrm{~b}$ ). Yet, the objective function given in Figure $5 \mathrm{~b}$ indicates a larger misfit. Imagine, compared with the linear velocity model, we have all but one of the 39 discrete vertical wavenumbers of the residual in place compared with having none in the case of the linear velocity medium, and yet the misfit is worse. If we look closely to the synthetic data, we realize that despite generating most of the reflections, they are shifted by about a half cycle, causing a larger misfit. This demonstrates the importance of getting the low wavenumbers of the model accurately in place prior to extracting the high wavenumbers - a well-known fact about FWI, but clearly emphasized by this example. Missing the higher model wavenumbers has smaller influence on the data, and we almost get a perfect fit, zeroobjective value at that end. Despite that the residual $(\Delta v)$ includes low wavenumbers that have larger magnitude, as shown in Figure 5a, which is generally expected, the response of the data to missing the high wavenumbers is even further muted by the nature of the source wavelet. Thus, the source wavelet acts as an additional filter at the high end of the wavenumber spectrum. As displayed, it is actually a direct filter with respect to the areas of velocity equal or higher than $3 \mathrm{~km} / \mathrm{s}$, the mapping velocity between frequency and wavenumber. Figure $5 \mathrm{c}$ shows the objective function (solid curve) corresponding to having only one of the 39 wavenumbers representing $\Delta v$ accurate, whereas the dashed straight horizontal line shows the objective function of the linear velocity model, both normalized by the highest value. Having only one accurate wavenumber produces a worse misfit than having none of the wavenumbers correct. This demonstrates the level of nonlinearity in FWI with respect to the velocity model. Interestingly, the intermediate wavenumbers produces the largest misfit when we have them right. Despite the complexity in attaining middle wavenumbers, they seemingly have a big influence on the data in the negative direction. So having accurate middle wavenumbers present in the model prior to getting the low wavenumbers in place can be detrimental.

\section{FILTERING THE GRADIENT}

We can control the wavenumbers admitted to the gradient using wavenumber filters or the scattering angle filter. The scattering angle filter provides a control that is optimal for the considered frequency and velocity. In other words, by allowing only large scattering angles to contribute, we are forcing wave path updates that include the resolution provided by the available frequencies. Such resolution, as equation 13 clearly demonstrates, is inversely proportional to the velocity. This is naturally expected because higher velocities admit waves with longer wavelengths, and thus less resolution. Scattering angle filtering explicitly honors these physical realities.
To access the scattering angle information, we naturally need to extend the gradient. Khalil et al. (2013) suggest a velocity normalized time lag $\zeta=(v(\mathbf{x}) \tau) / 4$, where $\tau$ is the conventional time-lag

a)

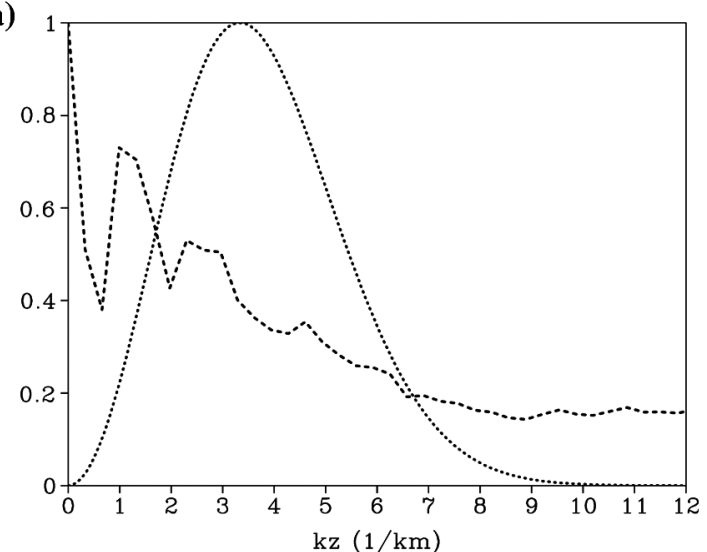

b)

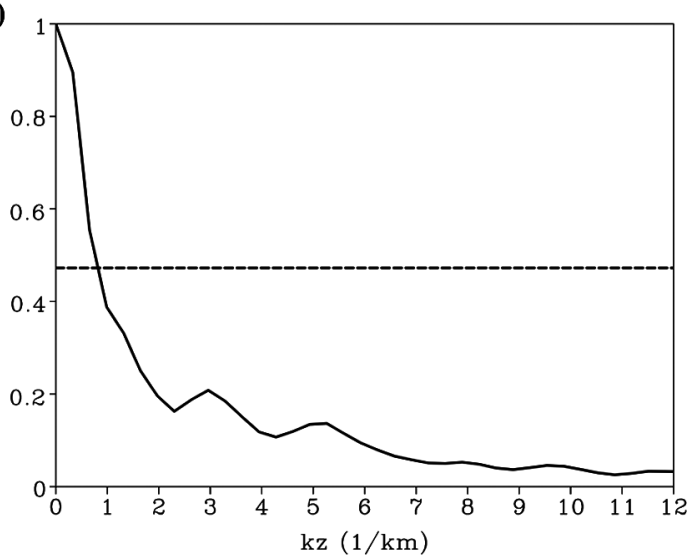

c)

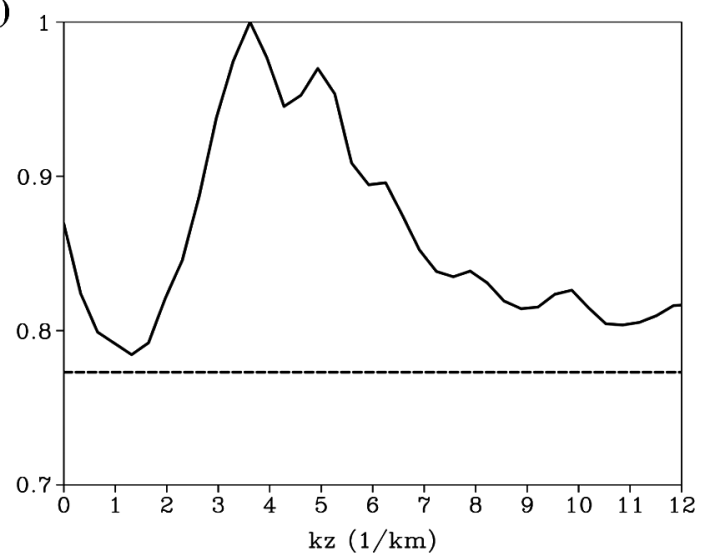

Figure 5. (a) The source wavelet spectrum mapped from frequency to wavenumber using a velocity of $3 \mathrm{~km} / \mathrm{s}$ (dotted curve), and the average vertical wavenumber spectrum of the velocity residual $\Delta v$ (dashed curve). (b) The objective function corresponding to a model with the residual missing the wavenumber given by the horizontal axis (solid). (c) The objective function corresponding to a model with the residual having only the wavenumber given by the horizontal axis (solid). In panels (b and c), the dashed straight line corresponds to the objective function value for the background velocity (Figure $3 b$ ). All vertical axes are normalized by the largest value. 
variable. Applying it to the gradients in equations 4 and 10 in the frequency domain yields (Alkhalifah, 2014b)

$$
\begin{aligned}
R_{\mathrm{FWI}}(\mathbf{x}, \zeta)= & \omega^{2} \sum_{i} u_{s_{i}}(\mathbf{x}) u_{r_{i}}(\mathbf{x}) e^{-4 i \omega \frac{\zeta}{v(\mathbf{x})}} \text { and } \\
R_{\mathrm{RWI}}(\mathbf{x}, \zeta)= & \omega^{2} \sum_{i}\left(u_{s_{i}}(\mathbf{x}) \delta u_{r_{i}}(\mathbf{x})\right. \\
& \left.+u_{r_{i}}(\mathbf{x}) \delta u_{s_{i}}(\mathbf{x})\right) e^{-4 i \omega \frac{\zeta}{v(\mathbf{x})}}
\end{aligned}
$$

With distance units, the modified time-lag extension admits a velocity-free relation between the scattering angle and the gradient wavenumber. Specifically, the scattering angle $\theta$ is then given by the following formula:

$$
\cos ^{2} \frac{\theta}{2}=\frac{|\mathbf{k}|^{2}}{k_{\zeta}^{2}},
$$

where $\mathbf{k}$ is the space wavenumber vector and $k_{\zeta}$ is the wavenumber (Fourier transform) corresponding to $\zeta$. As a result, we can use the fast Fourier transform to map to angle gathers, as the mapping equation 15 is free of spatial dependency. However, instead, we use equation 15 to filter out energy corresponding to low scattering angle $\theta$, and sum directly the rest over $k_{\zeta}$ (the zero $\zeta$ imaging condition). Finally, we inverse Fourier transform the gradient back to space.

Because $k_{\zeta}=(\omega / v)$, we can insert it directly into equation 13 to obtain

$$
\mathbf{k}_{m}=2 k_{\zeta} \cos \frac{\theta}{2} \mathbf{n},
$$

which shows that $k_{\zeta}$ provides control of the model wavenumber regardless of frequency.

\section{THE SCATTERING ANGLE WAVENUMBER CONTINUATION}

The scattering angle filter, as described above, offers an opportunity to control the wavenumbers we inject into the model from the gradients. It is not a direct control, such as a simple filter, it is, however, tied to the background velocity providing more

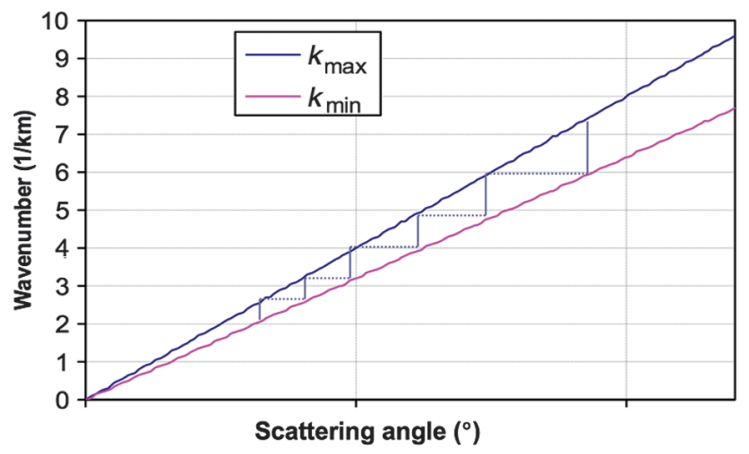

Figure 6. A schematic plot depicting the progress in scattering angle from high to low to allow for a proper wavenumber continuation. smoothness at higher background velocities considering the longer wavelengths and less smoothness at lower velocities. A schematic plot of wavenumber continuation based on scattering angle filtering regardless of frequency is shown in Figure 6, following in the footsteps of Sirgue and Pratt (2004) for frequencies.

Using the Marmousi velocity model, and specifically the residuals established in the earlier example, $\Delta v(x, z)=v(x, z)-v_{0}(z)$ (Figure $3 \mathrm{a}$ and $3 \mathrm{~b}$ ), we look at the least-squares fit objective as the function of a continuation in wavenumber contribution from low to high. I compare that with a similar continuation using scattering angles from large to small. I map the wavenumber to scattering angle using a constant velocity, and thus, can plot them both in Figure 7 for comparison. Despite the use of the exact velocity residual as gradient, the wavenumber continuation offers a local minimum in the center at approximately a $100^{\circ}$ scattering angle. Such local minimum is not present in the scattering angle continuation. The objective function for the background, linear velocity increase, and model (normalized) is one.

To understand this phenomenon, I compare the resulting velocity models from adding the velocity residuals with the background velocity through wavenumber continuation and scattering angle filtering. I will label both using the equivalent scattering angle for a constant velocity, so we can compare them at the same level. Figure $8 \mathrm{a}$ shows a vertical profile of the scattering-angle-filtered residual added to the linearly increasing background velocity model (dashed curve) along with the true Marmousi velocity at location $5.2 \mathrm{~km}$ (solid curve). In this case, we have effectively dropped the higher wavenumbers, but such a drop is background velocity dependent. If we compare this with an equivalent plot (Figure 8b), in which the filtering is done using pure wavenumber cutoff (dashed curve), we notice that the two curves have minor, but critical, differences. While for the scattering angle filtering, we have a better fit to the Marmousi actual values (solid curve) up shallow, due to higher resolution, we have a better fit (and higher resolution) for the wavenumber filtering at depth. However, considering that seismic velocity inversion tend to favor a top-to-bottom approach, having higher resolution in the shallow part provided by scattering angle

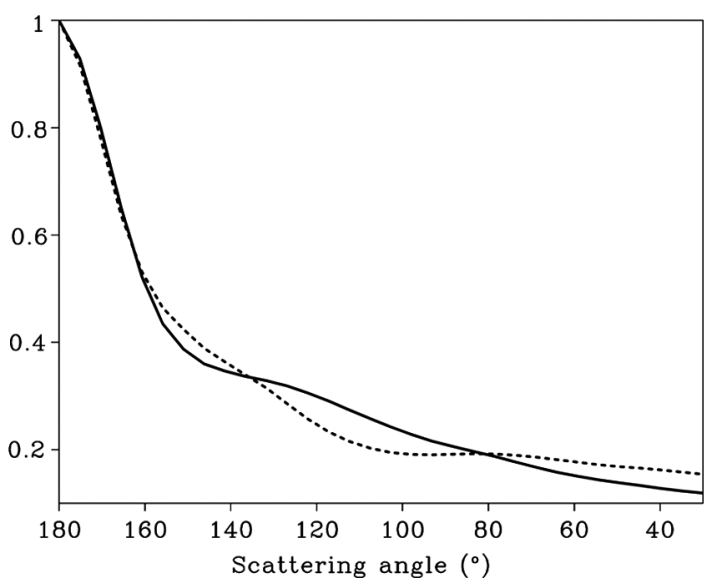

Figure 7. The objective function as a function of scattering angle for a continuation in terms of scattering angle from diving waves $\left(180^{\circ}\right)$ to reflections (solid curve) compared with a continuation in terms of wavenumber filtering from low to high mapped to scattering angle for comparison (dashed curve). 
filtering improves convergence and provides a more natural buildup. As a result, with scattering angle filtering, we avoided local minima to the continuation as apparent in Figure 7.

\section{THE SEG2014 BLIND TEST EXAMPLE}

The SEG2014 data set was built by Chevron and distributed without the true model as a test for FWI, in which the results were discussed in a workshop at the SEG annual meeting in 2014. In addition to the data, we were provided with an initial velocity model that captures accurately the topography of the sea bottom and is laterally homogeneous below the sea bottom as shown in Figure 9a. The initial velocity model also sets for us the model region that extends $6 \mathrm{~km}$ deep. We were, also, provided the true velocity at one lateral location extending to a depth of $1-2.4 \mathrm{~km}$, resembling information we may have from a well. We use this well velocity here only to check the accuracy of the inverted velocity models at that location.

Here, we invert for the model for frequencies exceeding $7 \mathrm{~Hz}$. In other words, we mute out the low frequencies to make the problem more realistic and to highlight the power of scattering angle filtering. A representative shot gather, in which low frequencies are muted, is shown in Figure 9b. The objective of FWI is to find the model that would produce shot gathers that fit those, such as the one in Figure 9b. We first focus on frequencies ranging from 7 to $8 \mathrm{~Hz}$ using a frequency-domain FWI (Pratt et al., 1996). This range, considering the noise in the data, provides the necessary signal-to-noise ratio capable of admitting plausible gradients. We use the scattering angle filter to administrate the wavenumber continuation for this frequency range. Starting from allowing just the large (more than $178^{\circ}$ ) scattering angle energy to contribute to the gradient and slowly relaxing the filter to include lower $\left(100^{\circ}\right)$ scattering angles in 50 iterations, we end up with the velocity model shown in Figure 10a. As expected, this velocity model is generally smooth considering the filtering; however, it includes many important features of the model. Allowing the smaller scattering angles (down to zero) to contribute, we end up with the velocity model in Figure 10b. Considering our starting frequency of $7 \mathrm{~Hz}$, the inversion managed to capture most of the features of the true velocity especially up
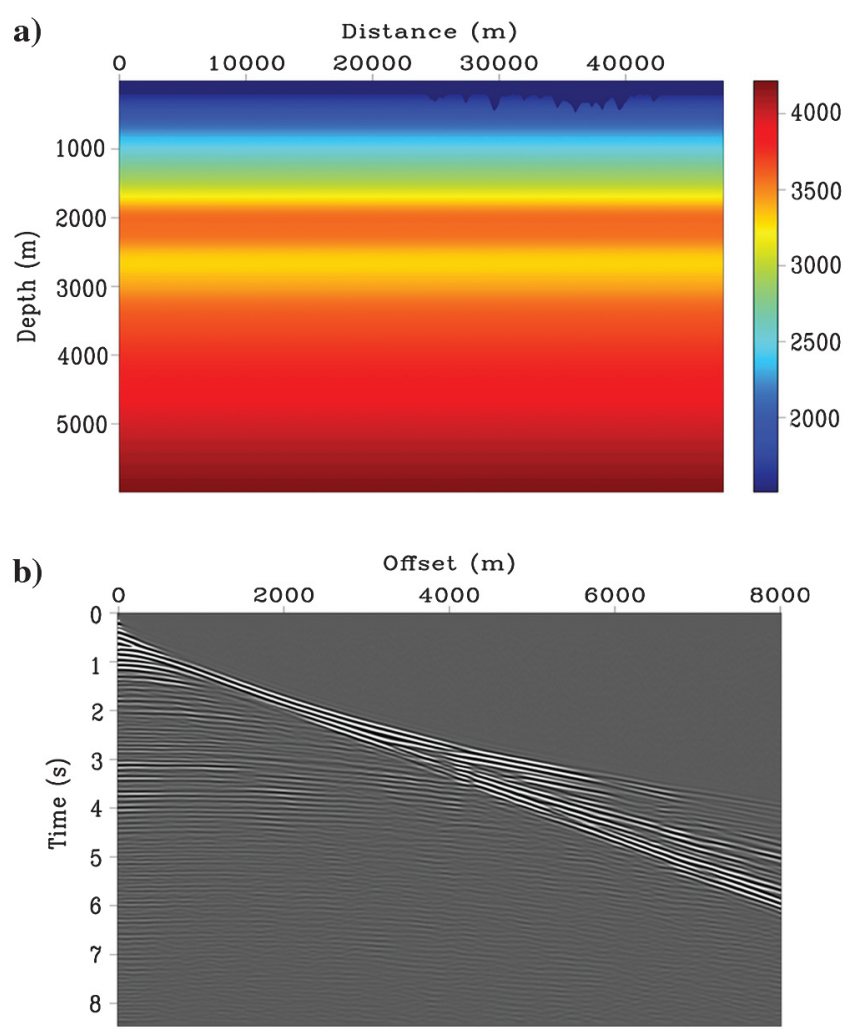

Figure 9. (a) The initial velocity model provided with the data set. (b) A representative shot gather from the SEG2014 blind test data set with frequencies less than $7 \mathrm{~Hz}$ muted.
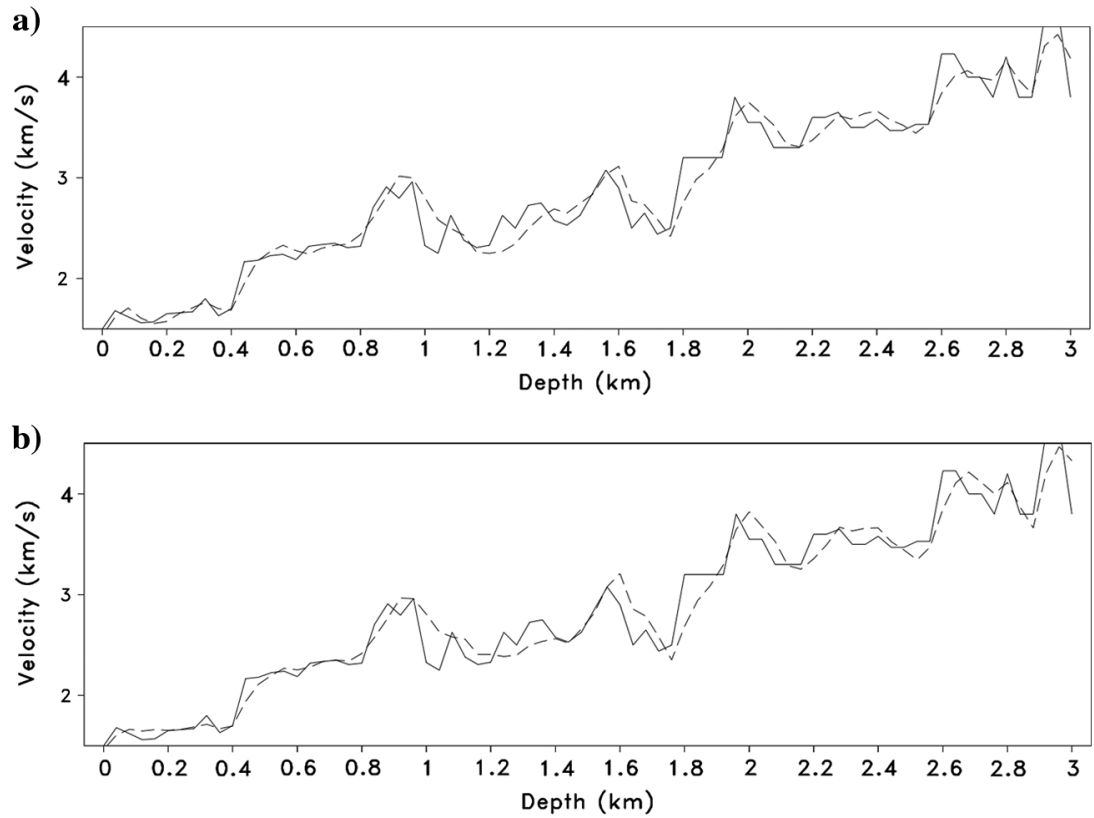

Figure 8. A velocity profile from the Marmousi at location $5.2 \mathrm{~km}$ (solid curves) compared with a smoothed residual added to the linearly increasing background velocity (dashed curves) with smoothing administrated using (a) scattering angle filtering and (b) wavenumber filtering at the same equivalent scattering angle. 
shallow. Comparison of a profile of this model with the provided true velocity at the location of the presumed well (Figure 10c) demonstrates that we ended up with a smooth version of the true model, at least at this location down to $2.4 \mathrm{~km}$ depth. Taking the inversion to higher frequencies $(13 \mathrm{~Hz})$ admits higher resolution velocity shown in Figure 11a, with a better fit to the well (Figure $11 \mathrm{~b}$ ). Using the inverted model to generate synthetic data allows us to compare it with the provided data as shown in Figure 12b. We have managed to reproduce most to the diving wave information accurately, as well as reflections down to approximately $4 \mathrm{~s}$. The scattering angle filter allowed us to build a reasonably accurate velocity model starting with a frequency of $7 \mathrm{~Hz}$. No MVA-based methods were used here.

\section{CONCLUSIONS}

The purpose of this study is to highlight the importance of recognizing the role of model wavenumbers in FWI and RWI and more importantly finding the proper approach to control such wavenumbers. This relatively short paper provides a summary of what can be extracted from analyzing the influence of the low and high wavenumbers in the data. A single low wavenumber missing from the
Figure 10. (a) The inverted velocity model after 50 iterations with gradients that include up to $100^{\circ}$ scattering angle energy. (b) The inverted velocity after an additional 50 iterations and allowing all scattering angles to contribute for the $7-8 \mathrm{~Hz}$ data. (c) A comparison with the provided true velocity at the presumed well location.
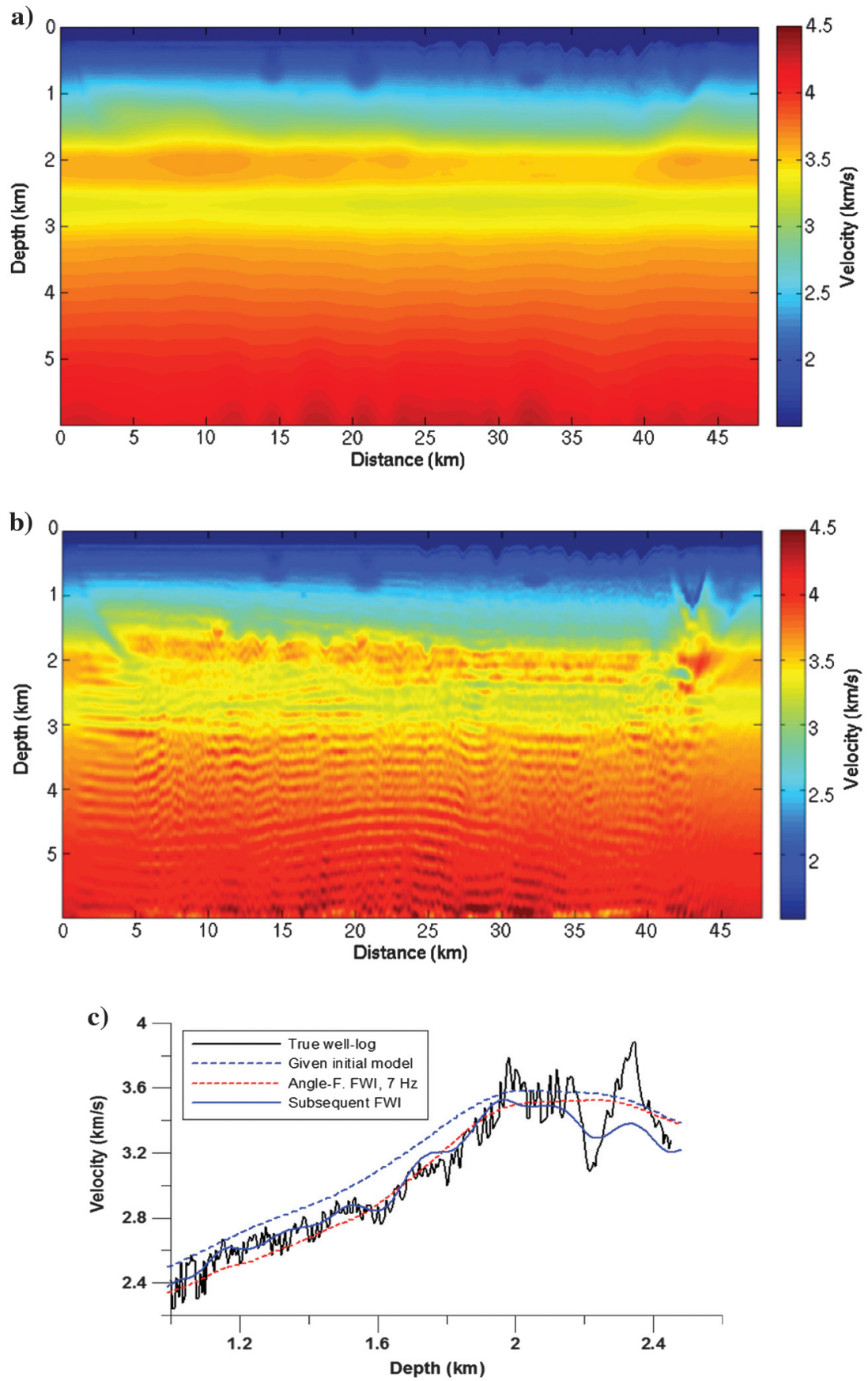
velocity residual is capable of inducing a larger misfit compared with even missing all the wavenumbers. A fact that could only further support the notion of wavenumber continuation. However, scattering angle continuation provides a better approach to orderly filling in the missing wavenumbers than common wavenumber filtering because the latter has its roots in the physical meaning of the scattering angle.
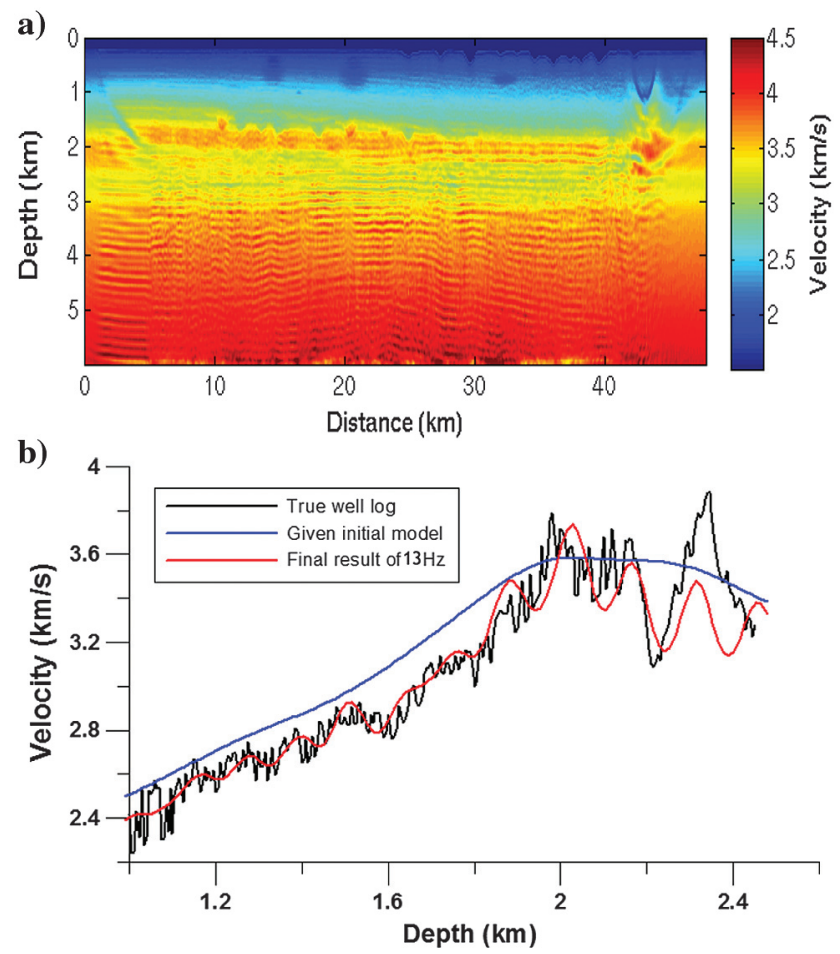

Figure 11. (a) The inverted velocity model after an additional 50 iterations with frequencies up to $13 \mathrm{~Hz}$. (b) A comparison with the provided true velocity at the presumed well location.

\section{ACKNOWLEDGMENTS}

I thank KAUST for its support. I am also grateful to $\mathrm{Z}$. Wu and Y. Choi for useful discussions and some of the numerical examples. I thank Chevron for the SEG2014 data set. I also thank the associate editor and the reviewers for their outstanding review of the paper.

\section{REFERENCES}

Albertin, U., G. Shan, and J. Washbourne, 2013, Gradient orthogonalization in adjoint scattering-series inversion: 83rd Annual International Meeting, SEG, Expanded Abstracts, 1058-1062.

Alkhalifah, T., 2014a, Research note: Full-waveform inversion of the unwrapped phase of a model: Geophysical Prospecting, 62, 397-403, doi: 10.1111/1365-2478.12089.

Alkhalifah, T., 2014b, Scattering-angle based filtering of the waveform inversion gradients: Geophysical Journal International, 200, 363-373, doi: 10.1093/gji/ggu379.

Alkhalifah, T., and Z. Wu, 2016, The natural combination of full and imagebased waveform inversion: Geophysical Prospecting, 64, 19-30, doi: 10 .1111/1365-2478.12264

Almomin, A., and B. Biondi, 2013, Tomographic full waveform inversion (TFWI) by successive linearizations and scale separations: 83rd Annua International Meeting, SEG, Expanded Abstracts, 1048-1052.

Al-Yahya, K., 1989, Velocity analysis by iterative profile migration: Geophysics, 54, 718-729, doi: 10.1190/1.1442699.

Bunks, C., F. Saleck, S. Zaleski, and G. Chavent, 1995, Multiscale seismic waveform inversion: Geophysics, 60, 1457-1473, doi: 10.1190/1 .1443880 .

Chavent, G., and R. Plessix, 1999, An optimal true-amplitude least-squares prestack depth-migration operator: Geophysics, 64, 508-515, doi: 10 1190/1.1444557.

Clement, F., G. Chavent, and S. Gmez, 2001, Migration based traveltime waveform inversion of 2-D simple structures: A synthetic example: Geophysics, 66, 845-860, doi: 10.1190/1.1444974.

Fleury, C., and F. Perrone, 2012, Bi-objective optimization for the inversion of seismic reflection data: Combined FWI and MVA: 82nd Annual International Meeting, SEG, Expanded Abstracts, doi: 10.1190/ segam2012-0797.1.

Jang, U., D. Min, and C. Shin, 2009, Comparison of scaling methods for waveform inversion: Geophysical Prospecting, 57, 49-59, doi: 10 $.1111 / \mathrm{j} .1365-2478.2008 .00739 . x$.

Jin, S., R. Madariaga, J. Virieux, and G. Lambar, 1992, Two-dimensional asymptotic iterative elastic inversion: Geophysical Journal International, 108, 575-588, doi: 10.1111/j.1365-246X.1992.tb04637.x.

Khalil, A., J. Sun, Y. Zhang, and G. Poole, 2013, RTM noise attenuation and image enhancement using time-shift gathers: 83rd Annual International Meeting, SEG, Expanded Abstracts, 3789-3793.

Ma, Y., D. Hale, B. Gong, and Z. Meng, 2012, Imageguided sparse-model full waveform inversion: Geophysics, 77, no. 4, R189-R198, doi: 10.1190/ geo2011-0395.1.
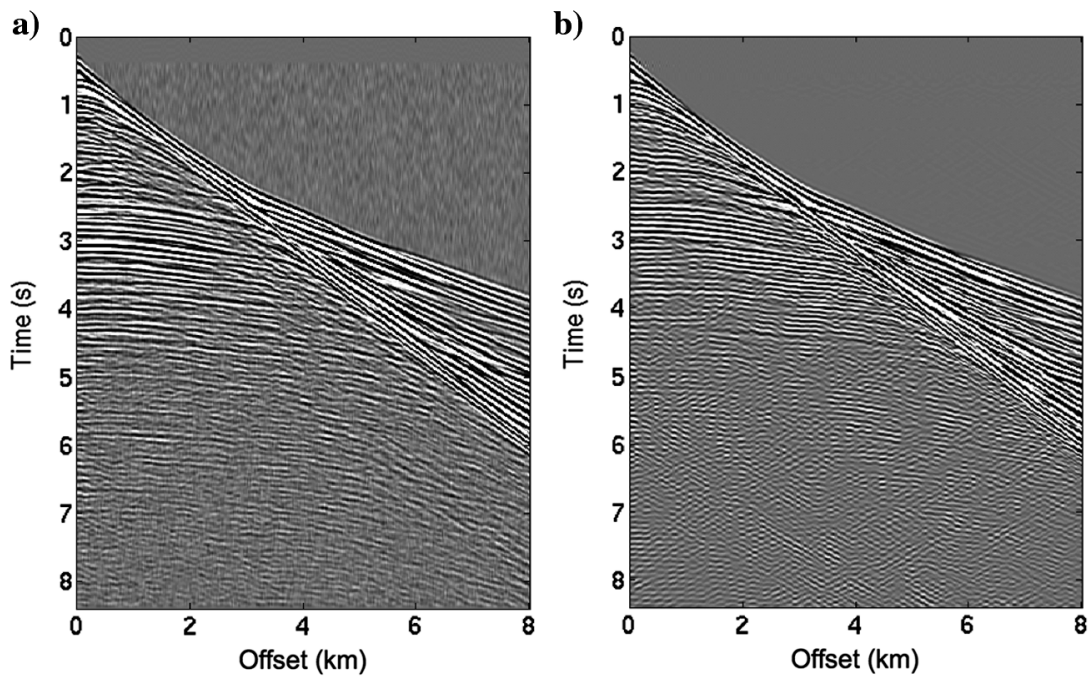

Miller, D., M. Oristaglio, and G. Beylkin, 1987, A new slant on seismic imaging: Migration and integral geometry: Geophysics, 52, 943-964, doi: 10.1190/1 .1442364 .

Plessix, R., Y. De Roeck, and G. Chavent, 1995 Automatic and simultaneous migration velocity analysis and waveform inversion of real data using a MBTT/ WKB J formulation: 65th Annual International Meeting, SEG, Expanded Abstracts, 1099-1102.

Pratt, R. G., Z.-M. Song, P. Williamson, and M. Warner, 1996, Two-dimensional velocity models from wideangle seismic data by wavefield inversion: Geophysical Journal International, 124, 323-340, doi: 10.1111/j .1365-246X.1996.tb07023.x.

Sirgue, L., and G. Pratt, 2004, Efficient waveform inversion and imaging: A strategy for selecting temporal frequencies: Geophysics, 69, 231-248, doi: 10.1190/ 1.1649391.

Symes, W., and M. Kern, 1992, Velocity inversion by differential semblance optimization for 2D common source data: 62nd Annual International Meeting, SEG, Expanded Abstracts, 1210-1213.

Tang, Y., S. Lee, A. Baumstein, and D. Hinkley, 2013, Tomographically enhanced full wavefield inversion: 83rd Annual International Meeting, SEG, Expanded Abstracts, 1037-1041.
Figure 12. (a) A shot gather from the original data. (b) A shot gather at the same location obtained by modeling the inverted velocity in Figure 11 a. 
Tarantola A. 1984, Inversion of seismic reflection data in the acoustic approximation: Geophysics, 49, 1259-1266, doi: 10.1190/1.1441754.

Thierry, P., S. Operto, and G. Lambare, 1999, Fast 2-D ray+Born migration/ inversion in complex media: Geophysics, 64, 162-181, doi: 10.1190/1 .1444513 .

Virieux, J., and S. Operto, 2009, An overview of full-waveform inversion in exploration geophysics: Geophysics, 74, no. 6, WCC1-WCC26, doi: 10 $.1190 / 1.3238367$
Wang, S., F. Chen, H. Zhang, and Y. Shen, 2013, Reflection-based full waveform inversion (RFWI) in the frequency domain: 83rd Annual International Meeting, SEG, Expanded Abstracts, 877881.

Xu, S., D. Wang, F. Chen, G. Lambare, and Y. Zhang, 2012, Inversion on reflected seismic wave: 82nd Annual International Meeting, SEG, Expanded Abstracts, doi: 10.1190/segam2012-1473.1. 